\title{
A Phylogenetic Analysis of Greek Isolates of Aspergillus Species Based on Morphology and Nuclear and Mitochondrial Gene Sequences
}

\author{
Antonios Krimitzas, ${ }^{1}$ Ioanna Pyrri, ${ }^{2}$ Vassili N. Kouvelis, ${ }^{1}$ \\ Evangelia Kapsanaki-Gotsi, ${ }^{2}$ and Milton A. Typas ${ }^{1}$ \\ ${ }^{1}$ Department of Genetics and Biotechnology, Faculty of Biology, National and Kapodistrian University of Athens, \\ Panepistemiopolis, 15701 Athens, Greece \\ ${ }^{2}$ Department of Ecology and Systematics, Faculty of Biology, National and Kapodistrian University of Athens, \\ Panepistemiopolis, 15784 Athens, Greece
}

Correspondence should be addressed to Milton A. Typas; matypas@biol.uoa.gr

Received 13 March 2013; Accepted 9 April 2013

Academic Editor: George Tsiamis

Copyright (c) 2013 Antonios Krimitzas et al. This is an open access article distributed under the Creative Commons Attribution License, which permits unrestricted use, distribution, and reproduction in any medium, provided the original work is properly cited.

Aspergillus species originating from Greece were examined by morphological and molecular criteria to explore the diversity of this genus. The phylogenetic relationships of these species were determined using sequences from the ITS and IGS region of the nuclear rRNA gene complex, two nuclear genes ( $\beta$-tubulin (benA) and RNA polymerase II second largest subunit $(r p b 2)$ ) and two mitochondrial genes (small rRNA subunit $(r n s)$ and cytochrome oxidase subunit I (coxl)) and, where available, related sequences from databases. The morphological characters of the anamorphs and teleomorphs, and the single gene phylogenetic trees, differentiated and placed the species examined in the well-supported sections of Aenei, Aspergillus, Bispori, Candidi, Circumdati, Clavati, Cremei, Flavi, Flavipedes, Fumigati, Nidulantes, Nigri, Restricti, Terrei, Usti, and Zonati, with few uncertainties. The combined use of the three commonly employed nuclear genes (benA, rpb2, and ITS), the IGS region, and two less often used mitochondrial gene sequences ( $r n s$ and $c o x 1)$ as a single unit resolved several taxonomic ambiguities. A phylogenetic tree was inferred using Neighbour-Joining, Maximum Parsimony, and Bayesian methods. The strains examined formed seven wellsupported clades within the genus Aspergillus. Altogether, the concatenated nuclear and mitochondrial sequences offer additional tools for an improved understanding of phylogenetic relationships within this genus.

\section{Introduction}

The genus Aspergillus is one of the most common and important genera of microfungi. Several species of the genus proved very useful cell factories for biotechnology purposes since they produce organic acids, extracellular enzymes, nutraceuticals (isoprenoids), and aroma compounds and are capable of fermenting various substrates under extreme cultivation conditions, usually intolerable to bacteria and yeasts [1-4]. Furthermore, the genus contains a number of species that produce toxic metabolites, act as opportunistic pathogens to humans, or decompose a wide variety of substrates $[5,6]$. Their high diversity combined with the worldwide distribution necessitates the detailed taxonomic and phylogenetic study of Aspergillus species in order to provide more appropriate tools for their accurate and fast identification.

The first most comprehensive taxonomic treatise of the genus was based on morphological criteria and included 150 taxa that were separated in "groups" [7]. These clusters of species ("groups") were later revised to 18 "sections" in 6 subgenera, in order to receive nomenclatural status [8]. In followup, a list of 182 species valid names was compiled [9], and expanded to $\sim 250$ species [3], and it is expected to increase further as new species are discovered and speciation concepts are refined [10]. 
During the last decade, a revision of the genus Aspergillus is in progress, as a result of molecular analyses which have offered a better insight into taxonomic and phylogenetic relations [10-13]. The revision is based on polyphasic approaches that include molecular data, as well as morphological, physiological and ecological characteristics. Although no single method worked flawlessly in recognising species, molecular data have largely supported previously inferred relationships that were based on the other characters [3]. Single locus DNA sequence studies were a common practice in fungi in the past, thus, large amounts of information have been accumulated in databases for several parts of the nuclear ribosomal rRNA repeat (in particular the ITS and 28S) [14-16] and genes like the $\beta$-tubulin [17]. The molecular identification of Aspergillus species is currently based on sequences from genes $\beta$-tubulin, calmodulin, actin, and ITS [5, 12, 13, 18-22]. A phylogenetic study of Aspergillus species, based on four single nuclear genes that were concatenated and used as one unit, attempted to resolve the existing taxonomic ambiguities and has resulted in a reevaluation of the genus Aspergillus $[23,24]$.

In other fungal genera, like Lecanicillium, Verticillium, and Beauveria, the use of mitochondrial (mt) genes and $\mathrm{mt}$ intergenic regions proved an extremely useful tool to reveal species differences within a genus and even helped to resolve taxonomic ambiguities (e.g., the small rRNA subunit ( $r n s)$, the NADH dehydrogenase subunits 1 (nad1) and 3 (nad3), the mt intergenic domains nad3-atp9 and the atp6rns) [25-27]. Similarly, other nuclear regions/genes like the RNA polymerase II largest subunit gene $(r p b 1)$ and the IGS region of the rRNA repeat also proved to be excellent tools for discriminating species within Metarhizium, Verticillium and Aspergillus [23, 27, 28].

In the present study the efficiency of combined nuclear and mitochondrial gene sequence data was evaluated for the identification of species that are distributed in most of Aspergillus sections. In detail, the commonly used (ITS1-5.8SITS2, RNA polymerase II second largest subunit gene $(r p b 2)$ and b-tubulin (benA)) and the less often exploited (IGS) nuclear regions were assessed along with two mitochondrial genes, cytochrome oxidase subunit I (coxl) and small rRNA subunit ( $r n s$ ), for the identification of Aspergillus species and the evaluation of their phylogenetic affinities. The objective of this work is to test hypotheses based on morphological criteria and data obtained from a multilocus DNA sequence analysis on the phylogeny within the genus Aspergillus.

\section{Materials and Methods}

Several strains of Aspergillus have been isolated from the indoor air of a food industry in Greece, as well as from food samples. Additional strains of representative Aspergillus spp. were studied from the ATHUM Culture Collection of Fungi in the University of Athens and ex-type cultures were obtained from the Agricultural Research Service ARS (NRRL) at Peoria, USA (kindly provided by Dr. S. Peterson). A total of thirty six strains belonging to thirty-one Aspergillus species were studied (Table 1). All strains are maintained in the ATHUM Culture Collection of Fungi.
The strains of Aspergillus were three-point inoculated in selective nutrient media and incubated on Czapek Yeast Autolysate Agar (CYA) at $25^{\circ} \mathrm{C}$ and $37^{\circ} \mathrm{C}$, on Malt Extract Agar (MEA) at $25^{\circ} \mathrm{C}$, and on $\mathrm{G} 25 \mathrm{~N}$ at $25^{\circ} \mathrm{C}$ [29]. The macromorphology of the colonies on each of the media, as well as the micromorphology on MEA, were studied after 7 days of incubation. Microscopic examination was performed by teasing apart the sample in a drop of $70 \%$ ethanol on a glass slide, a coverslip was placed on it, and it was observed under a Zeiss microscope in plain light or Differential Interference Contrast. The photographs were taken with an Axiocam digital camera (Zeiss, Germany). When the evaluation of the morphological data was completed, all strains were further examined with molecular markers and the data was used in phylogenetic analyses. Additional taxa were included to represent most of the sections recognized in the genus Aspergillus.

Mycelium preparation and fungal DNA extraction procedures used are previously described [30]. PCR primers used in the amplification reactions for the ITS1-5.8S-ITS2 region were 18S-ITS1 and 28S-ITS2 [27] and for the IGS region were CNL12 and CNS1 [31]. For the nuclear genes and specifically for the RNA polymerase II second largest subunit gene $(r p b 2)$, the primers used were RPB2-6F and RPB2-7R [32] and for the $\beta$-tubulin gene (ben $\mathrm{A})$ the primers were bt2a and bt2b [33]. Primers for the amplification of $\mathrm{mt}$ genes were NMS1 and NMS2 for rns [34] and cox1F and cox1R for cox1 [35]. The PCR amplicons were sequenced using a Thermo Sequenase Primer Cycle Sequencing kit (Amersham Biosciences, Amersham, UK), with a LICOR 4200 IR2 (LICOR, Lincoln NE, USA) automated sequencer. All fragments were read in both directions and nucleotide sequences were submitted to GenBank database (Table 1).

Maximum parsimony (MP), Neighbour-Joining (NJ), and Bayesian inference (BI) analyses were employed in order to create the phylogenetic trees. MP, and NJ analyses of nucleotide datasets were performed with PAUP ${ }^{*} 4.0 \mathrm{~b} 10$ [36] using 1,000 and 10,000 replicates, respectively, with random addition of taxa and tree-bisection reconnection branch swapping. Reliability of nodes was assessed using 1,000 and 100 bootstrap iterations (for NJ and MP analyses, resp.) and for the NJ analyses the Kimura-2 parameter model was employed. The BI was performed with MrBayes v3.1 [37]. A gamma distribution model of site variation was used, calculated with PAML [38]. For datasets ITS1-5.8S-ITS2, IGS, benA, rpb2, rns, cox1 and the concatenated data, alpha $(\alpha)$ was $0.470,0.630,0.540,0.400,0.348,0.527$, and 0.403, respectively. Random starting trees were used and the burnin period was 1,000,000 cycles, as this was found to be clearly sufficient for the likelihood and the model parameters to reach equilibrium. After the burn-in, 1,000 trees were sampled every 100 cycles during the sampling period $(1,000,000$ cycles). Two independent MCMCMC searches were run for each dataset using different random starting points. The hypocrealean fungus Metarhizium anisopliae from the Class Sordariomycetes, a close relative to Eurotiomycetes, was used as outgroup in all datasets. When the species Coccidioides immitis from Onygenales, an order within Eurotiomycetes, was alternatively employed, the tree topologies from all 


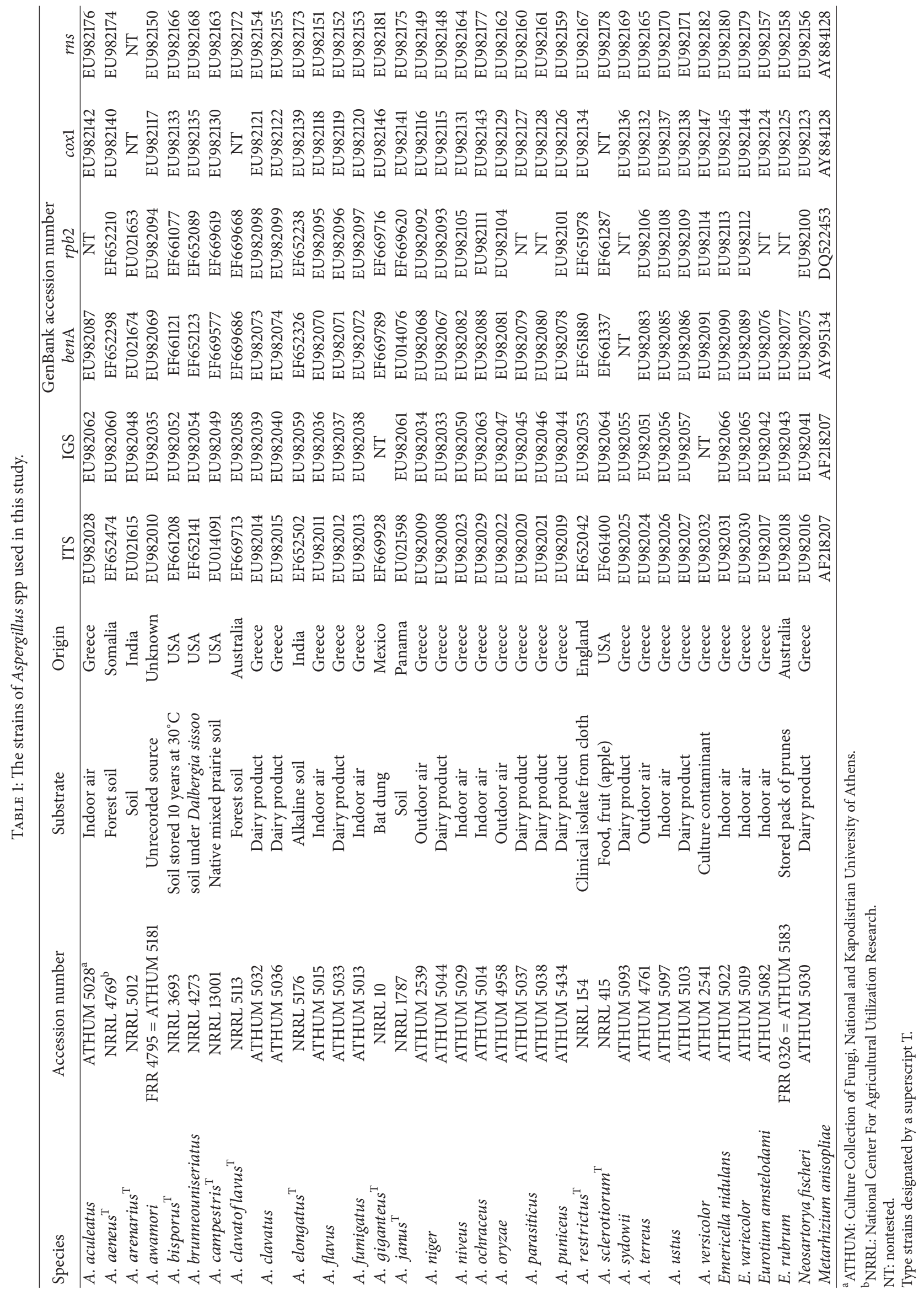


TABle 2: Phenotypic characters of Aspergillus species originating from Greece.

\begin{tabular}{lccccccc}
\hline Species & ATHUM number Vesicle shape & Vesicles $(\mu \mathrm{m})$ & Metulae $(\mu \mathrm{m})$ & Phialides $(\mu \mathrm{m})$ & \multicolumn{2}{c}{ Conidia } \\
& & & & & \\
Ornamentation
\end{tabular}

analyses were identical (data not shown). All sequences of Aspergillus species and their teleomorphs not amplified in this study were taken from GenBank and are provided with their Accession Numbers and the species names provided by the database. Aligned matrices are available in Treebase, http://purl.org/phylo/treebase/phylows/study/12165.

\section{Results}

3.1. Morphological Analysis. The cultural characteristics of the Aspergillus spp. have been recorded for all the strains and their evaluation was critical in cases of closely related species. The colony texture, colour and growth rate on the nutrient media, the formation of exudates, soluble pigments and sclerotia, and the thermotolerance were examined. The most important diagnostic characters are the morphological features of the conidial heads, including their growth pattern, size and seriation, dimensions and surface texture of the conidiophore, the shape and size of the metulae, phialides and conidia, and the colour and the wall ornamentation of the phialidospores (Figure 1). The range of variability found for the main morphological characteristics of the strains of Aspergillus spp. originating from Greece is presented in Table 2. In addition, the morphology of the teleomorph has been examined in detail when the holomorph was present. There is a great morphological variation in the type of ascomata, from loosely interwoven hyphae to compact sclerotioid structures, as well as in the size, ornamentation, and sculpture of the ascospores.

3.2. DNA Analyses. Amplified parts of genes and regions had variable sizes (i.e., in bp, 478-532 for ITS, 650-850 for IGS, 411-587 for benA, 785-811 for $r p b 2,536-555$ for $r n s$ and 805-847 for cox1), reflecting to the apparent genetic variability between different Aspergillus species. Amplicons were sequenced in both directions, sequences were combined and compared with all known related sequences in the databanks, and appropriate phylogenetic trees were constructed. Phylogenetic trees deduced from NJ analyses were drawn (Figures 2, 3, 4, 5, and 6), and parsimony and Bayesian methods were used to examine the sensitivity of the resulting trees and tree topologies. Trees remained largely invariant to these manipulations, and topologies were similar for each gene tested independently of the phylogenetic method applied.

The phylogenetic analysis of ITS and benA sequences included 227 and 257 taxa, respectively, belonging to 210 and 235 species of Aspergillus and their teleomorphs (i.e., Emericella, Eurotium, Fenellia, Neocarpenteles, Neosartorya, Neopetromyces, Petromyces, and Warcupiella), in order to determine their phylogenetic positions (Figures 2 and 3). For the datasets of IGS (Supplemental Figure S1 of Supplementary Material available online at http://dx.doi.org/10.1155/2013/ 260395) and cox1 (Supplemental Figure S2) the sequences analysed were significantly fewer than above, since there are no other entries in the databanks apart from those examined in our work (Table 1). Finally, the datasets of $r p b 2$ (Figure 4) and rns (Figure 5) were enriched with the addition of 147 and 10 more species, respectively, from databanks.

The ITS dataset included 634 characters, 376 of which were parsimony informative, resulting to 10 parsimonious trees (tree length: 2,606, consistency index (CI): 0.360 and retention index (RI): 0.824). Among the 790 polymorphic sites of the ben A sequences, 533 were phylogenetically informative. The topology of the NJ tree is the same as one of the 9,980 most parsimonious trees inferred by the PAUP programme (length: 7,986 steps, CI: 0.183 , and RI: 0.754 ). The 


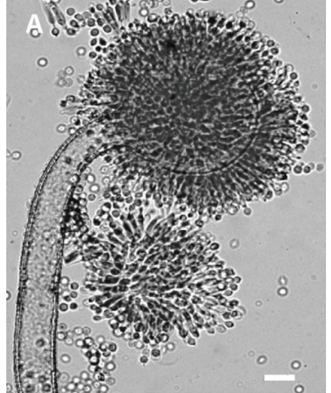

(a)

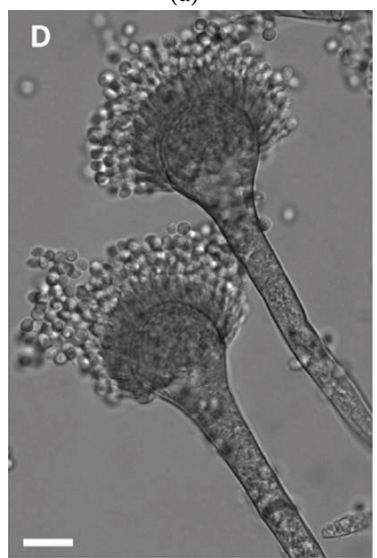

(d)

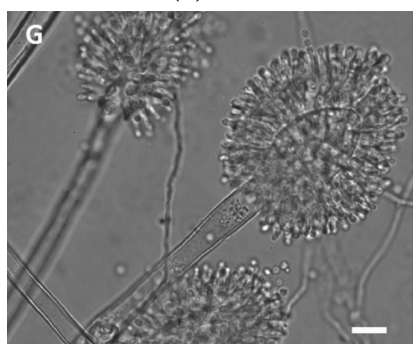

(g)

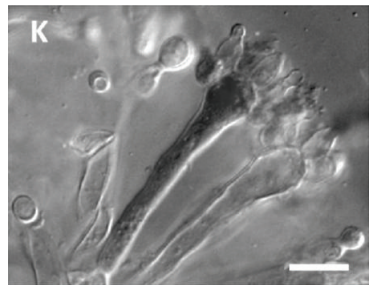

(k)

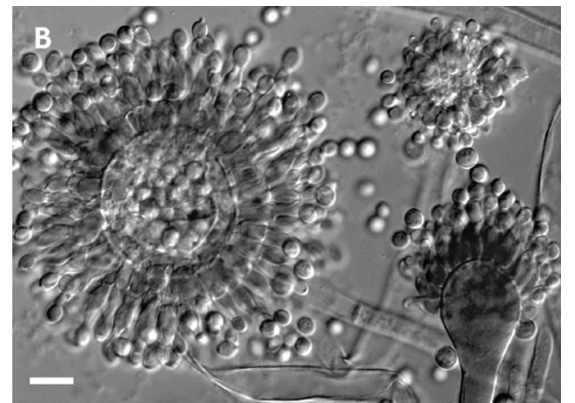

(b)

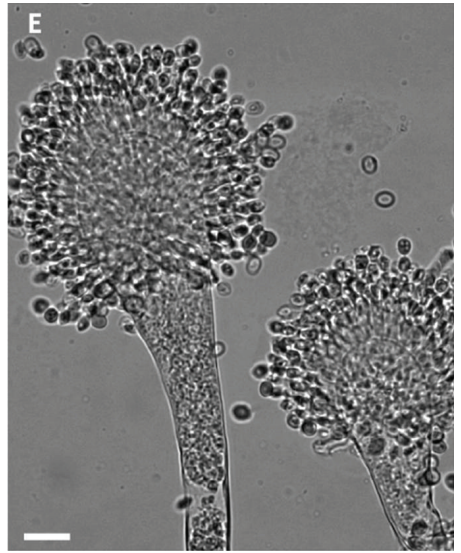

(e)

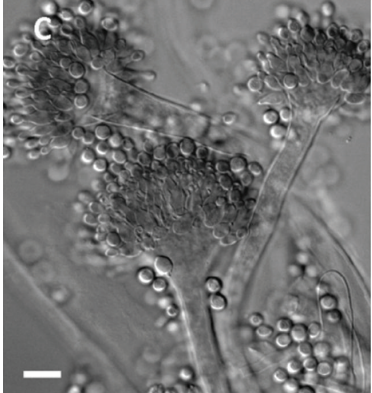

(c)

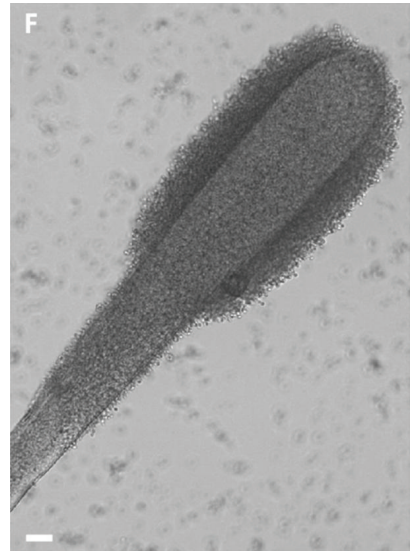

(f)

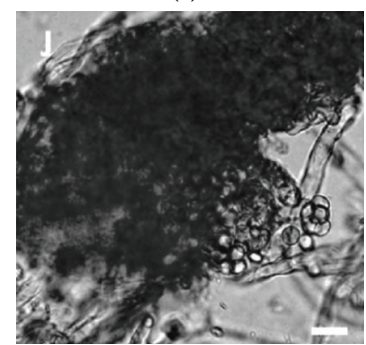

(j)

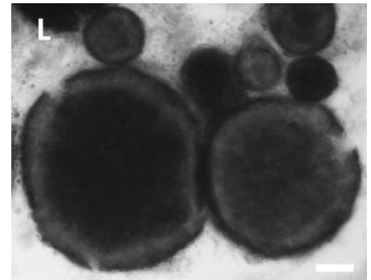

(1)

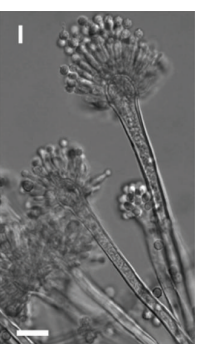

(i)

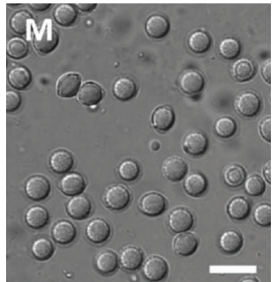

(m)

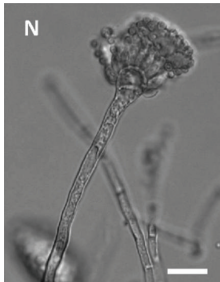

(n)

FIgURE 1: Anamorphic and teleomorphic characters of Aspergillus spp. (a) Aspergillus ochraceus ATHUM 5014. (b) Aspergillus flavus ATHUM 5033. (c) Aspergillus parasiticus ATHUM 5037. (d) Aspergillus fumigatus ATHUM 5013. (e) Aspergillus clavatus ATHUM 5036. (f) Aspergillus giganteus NRRL 10. (g) Aspergillus janus NRRL 1787. (h) Aspergillus versicolor ATHUM 2541. (i) Aspergillus sydowii ATHUM 5093. ((j)(k)) Eurotium amstelodami ATHUM 5082. ((l)-(n)) Neosartorya fischeri ATHUM 5030. ((a)-(i), (k), (n)) Conidial heads with phialides and conidia. (j) Part of cleistothecium with asci and ascospores. (l) Cleistothecia. (m) Ascospores. Bars = ((a) $-(\mathrm{e}),(\mathrm{g})-(\mathrm{n})) 10 \mu \mathrm{m}$; (f) $20 \mu \mathrm{m}$; (l) $50 \mu \mathrm{m}$.

rpb2 and cox1 dataset included 1,140 and 908 characters, with 655 and 303 parsimony informative characters, and produced 20 and 1,185 equally parsimonious trees (tree length: 8,927 and 1,185, CI: 0.152 and 0.594, and RI: 0.690 and 0.670 ), respectively. The IGS dataset included 901 characters, with 735 parsimony informative characters (tree length: 5,829,
CI: 0.354, and RI: 0.516). Finally, the rns dataset included 614 characters, with 130 parsimony informative characters (tree length: 512, CI: 0.534, and RI: 0.731).

Thirty-six strains studied in this work belong to 31 Aspergillus species (see Table 1). ITS sequences from these strains, together with sequences from other species of the 


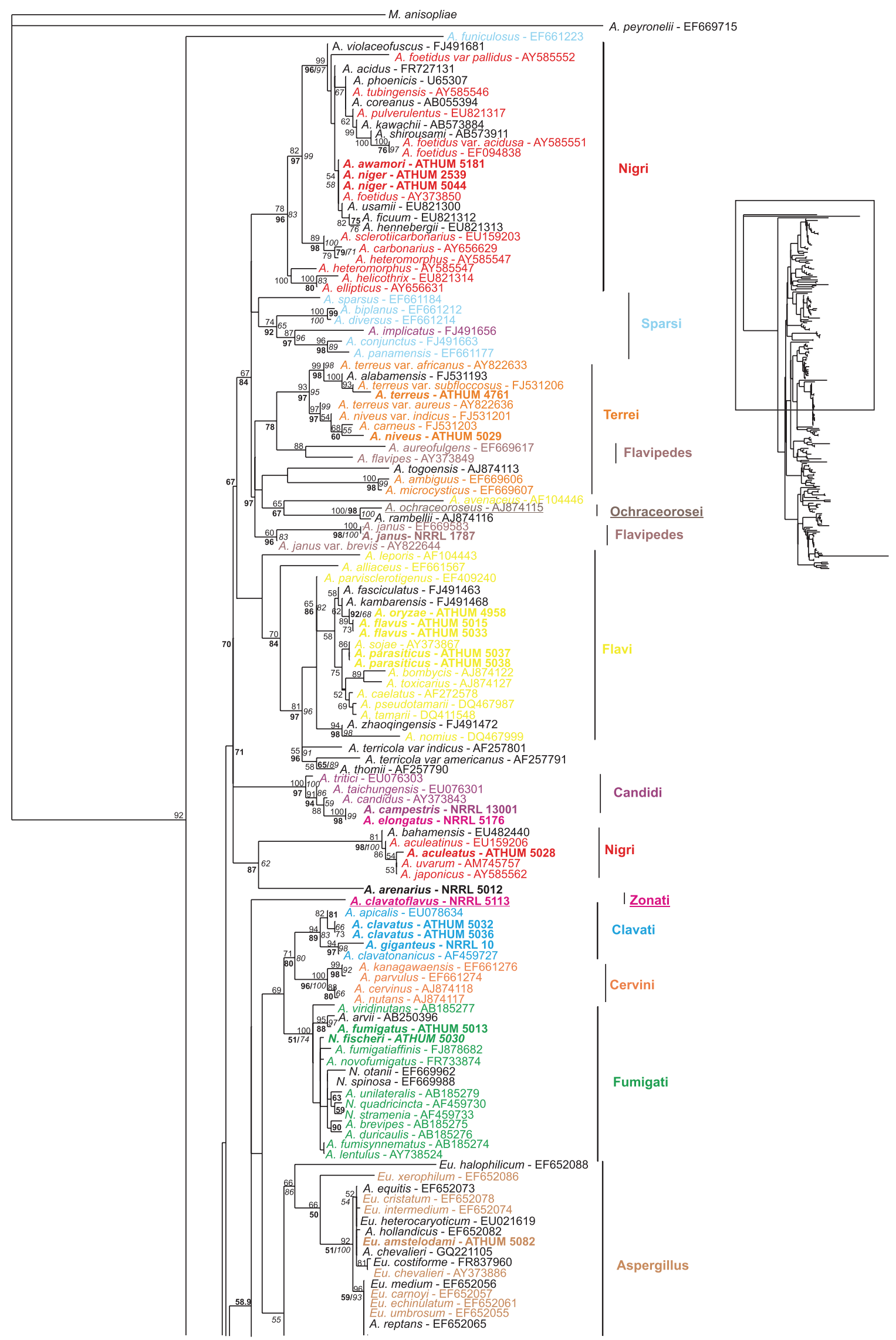

(a)

FIgUre 2: Continued. 


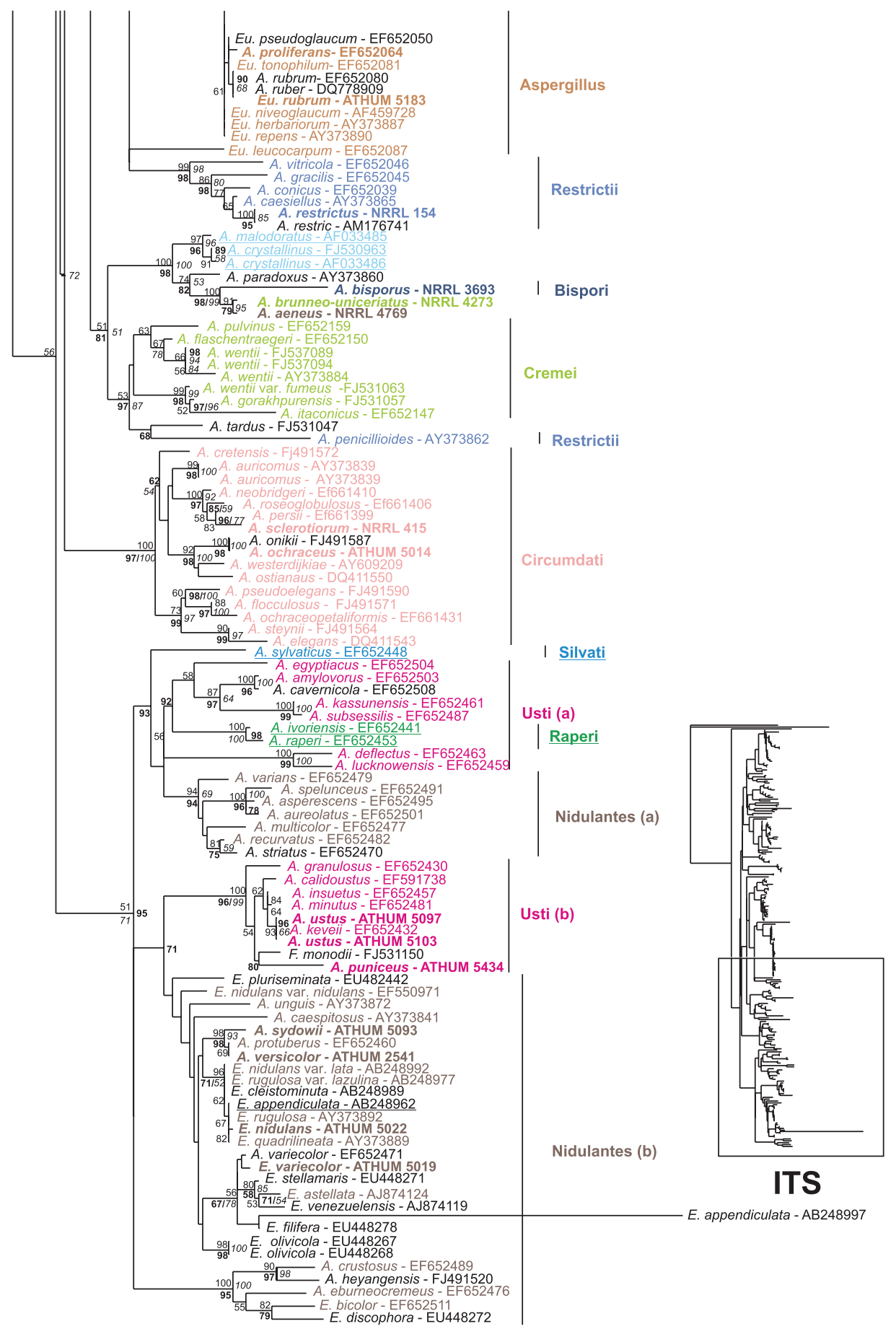

0.1

Clavati Fumigati Flavi Usti Candidi Nigri Terrei Circumdati Nidulantes Restrictii Sparsi

Zonati Silvati Flavipedes Aspergillus Cervini Bispori Cremei Ochraceorosei Raperi

(b)

Figure 2: Phylogenetic tree constructed from unambiguously aligned DNA sequences of the ITS domain as produced by NJ. Sequences obtained during this study are presented in bold, whereas those retrieved from GenBank are shown in roman with their Accession Numbers. Clade credibility using NJ calculated from 1,000 replicates (numbers in roman), parsimonial BS support calculated from 100 replicates (numbers in italics) using PAUP, and PPs produced by 1,000,000 generations (numbers in bold) using MrBayes are shown. 


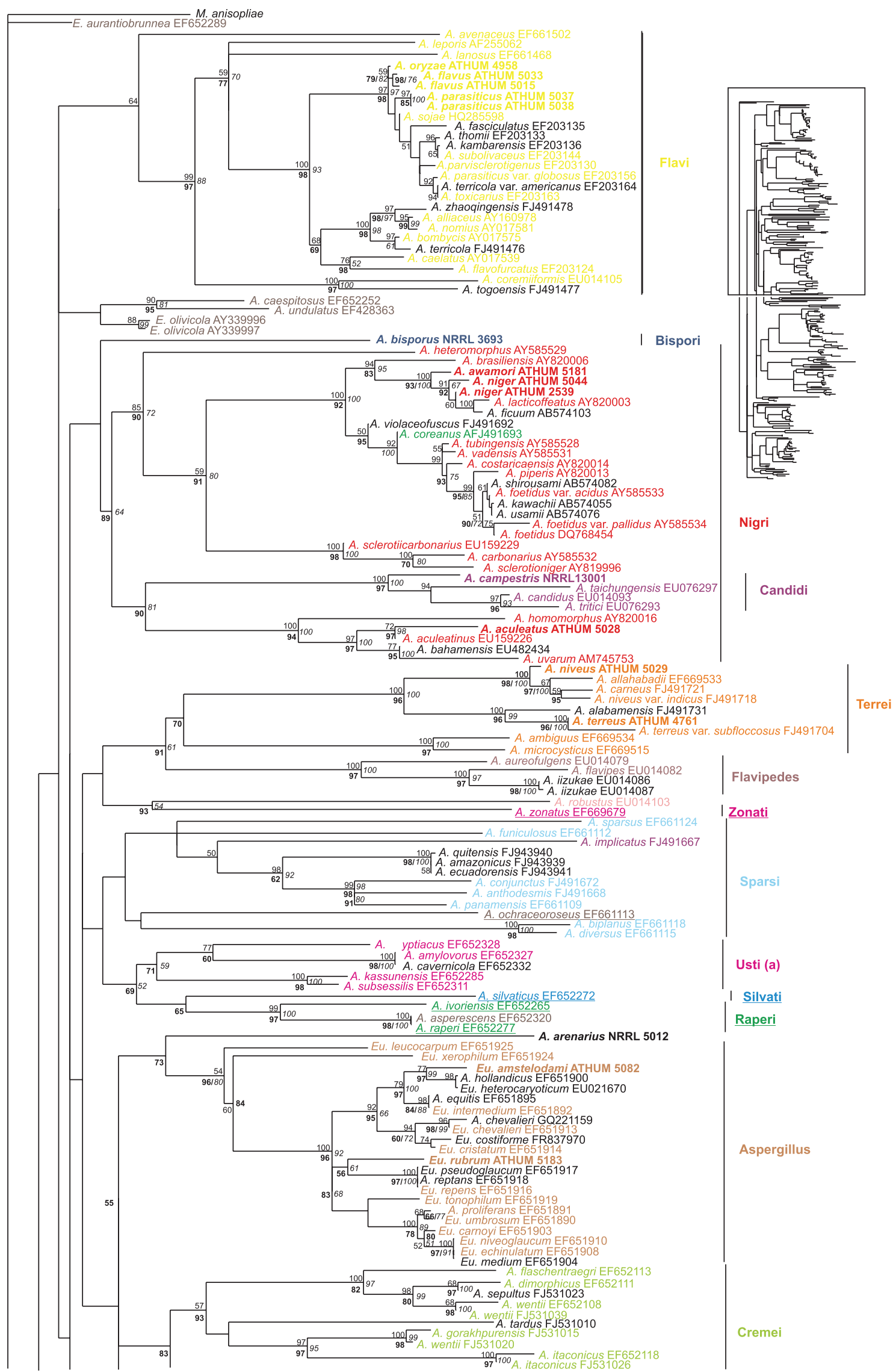

(a)

FIgUre 3: Continued. 


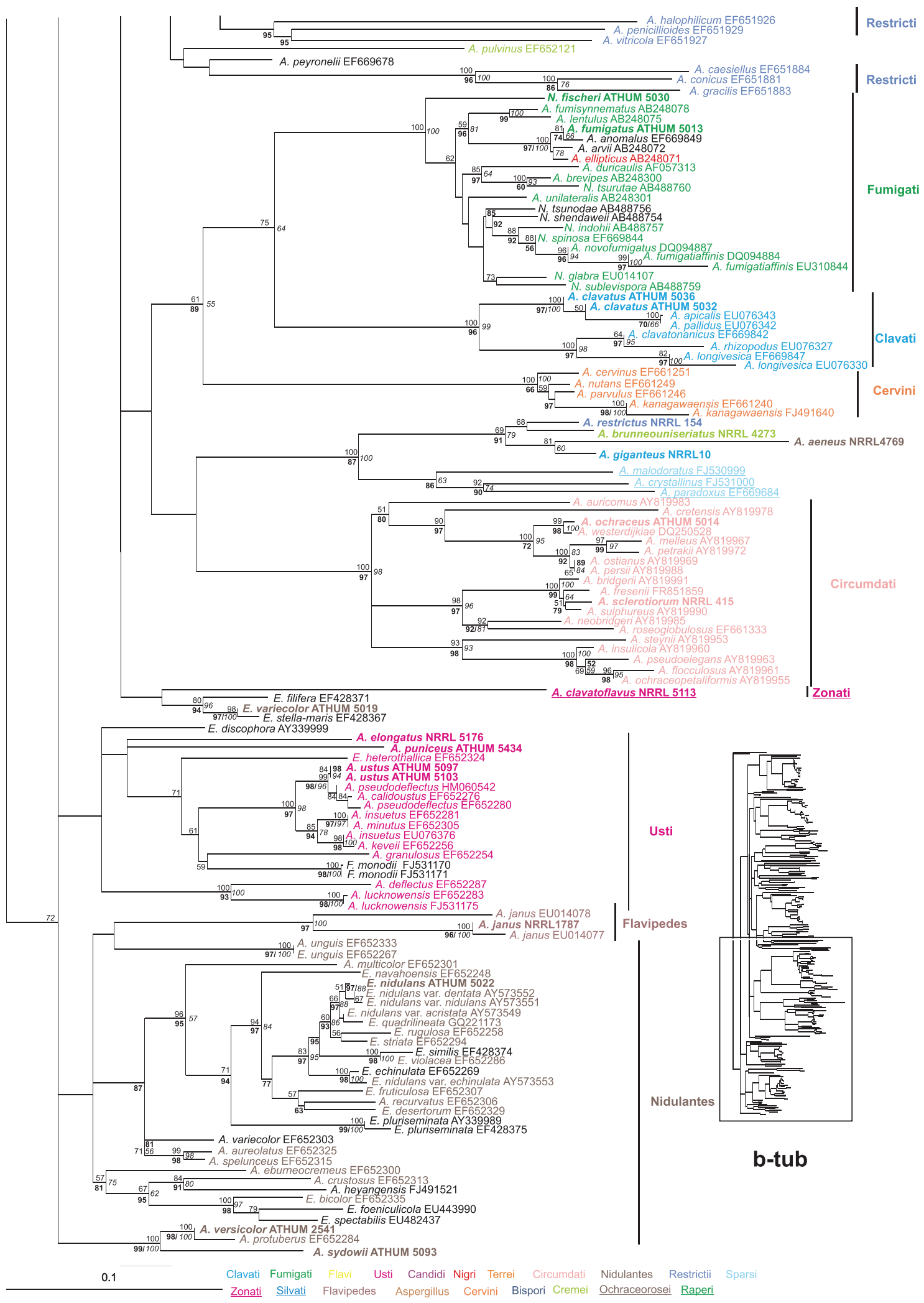

(b)

FIgURE 3: Phylogenetic tree constructed from unambiguously aligned combined DNA sequences of the benA gene as produced by NJ. Sequences obtained during this study are presented in bold, whereas those retrieved from GenBank are shown in roman with their Accession Numbers. Clade credibility using NJ calculated from 1,000 replicates (numbers in roman), parsimonial BS support calculated from 100 replicates (numbers in italics) using PAUP, and PPs produced by 1,000,000 generations (numbers in bold) using MrBayes are shown. 


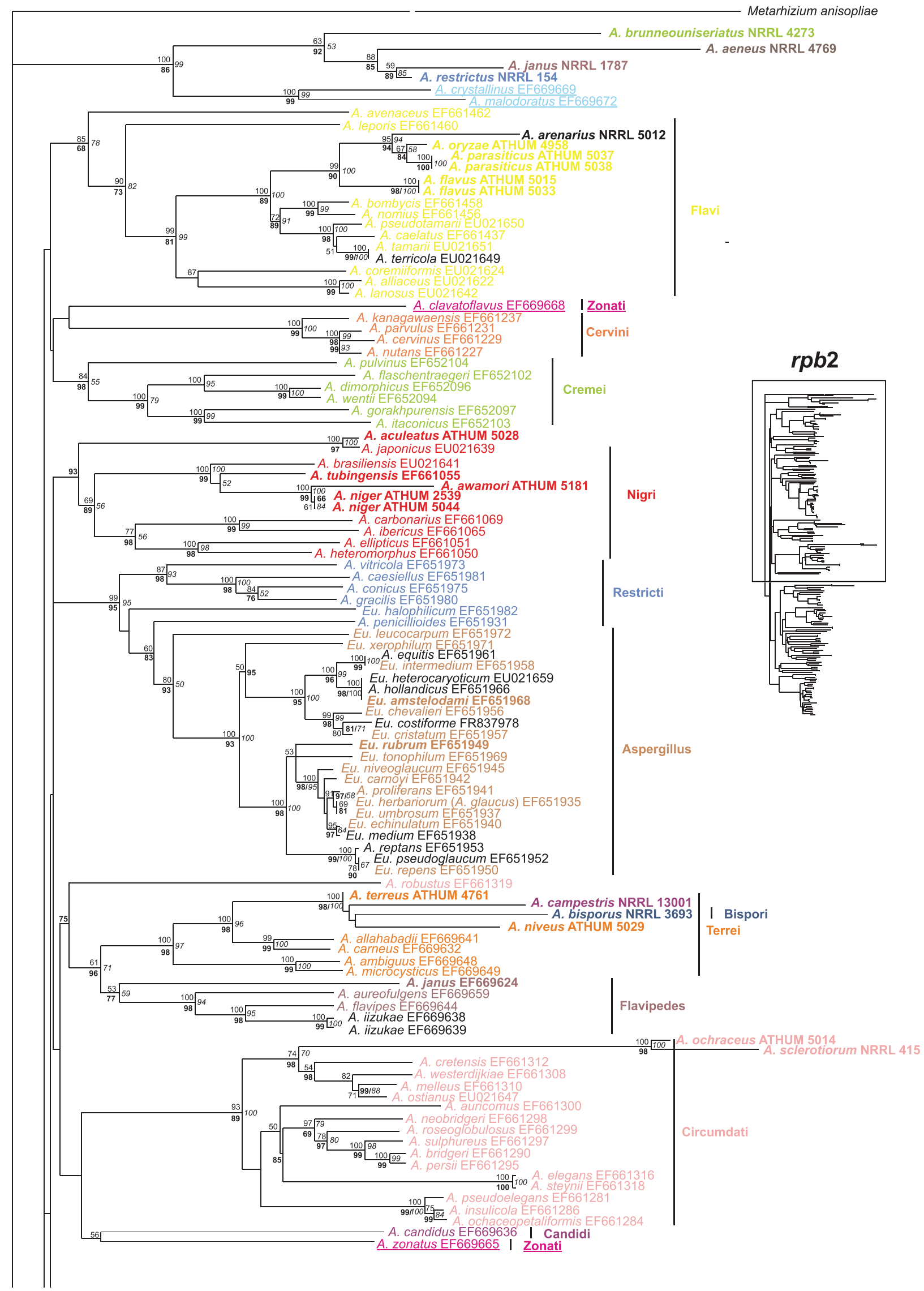

(a)

FIGURE 4: Continued. 


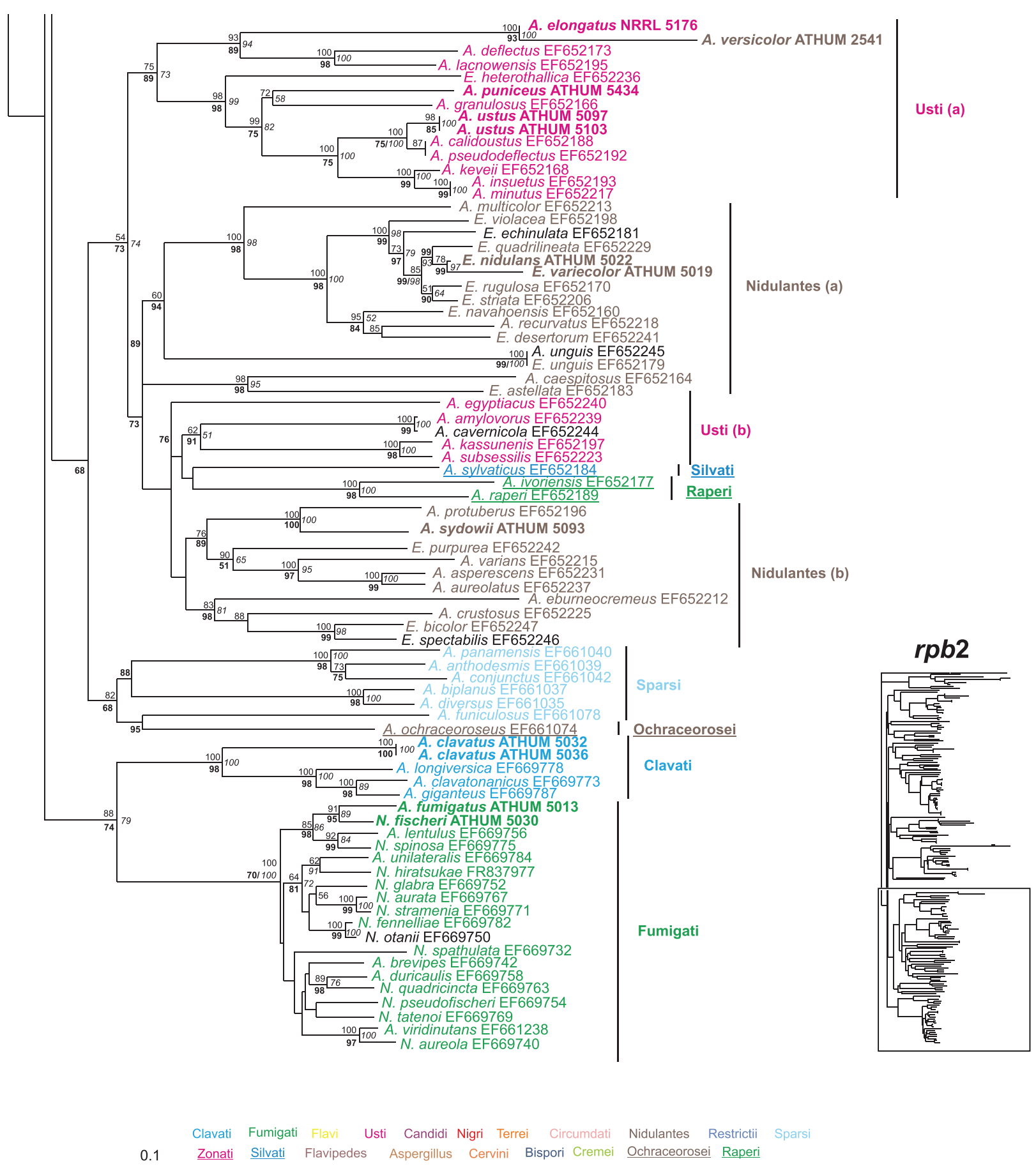

(b)

FIGURE 4: Phylogenetic tree constructed from unambiguously aligned combined DNA sequences of the nuclear $r p b 2$ gene as produced by NJ. Sequences obtained during this study are presented in bold, whereas those retrieved from GenBank are shown in roman with their Accession Numbers. Clade credibility using NJ calculated from 1000 replicates (numbers in roman), parsimonial BS support calculated from 100 replicates (numbers in italics) using PAUP, and PPs produced by 1,000,000 generations (numbers in bold) using MrBayes are shown.

genus that were retrieved from the GenBank (altogether around 200), clustered well within their corresponding sections of genus Aspergillus (Figure 2). The NJ, MP bootstrap, and Posterior Probabilities (PPs) support of all sections ranged from 51-100\%. Members of Candidi, Cervini, and Circumdati clustered with $100 \%$ and likewise Raperi, and Restricti showed higher than $90 \%$ support for all three methods. Species of sections Aspergillus (Eurotium xerophilum and 


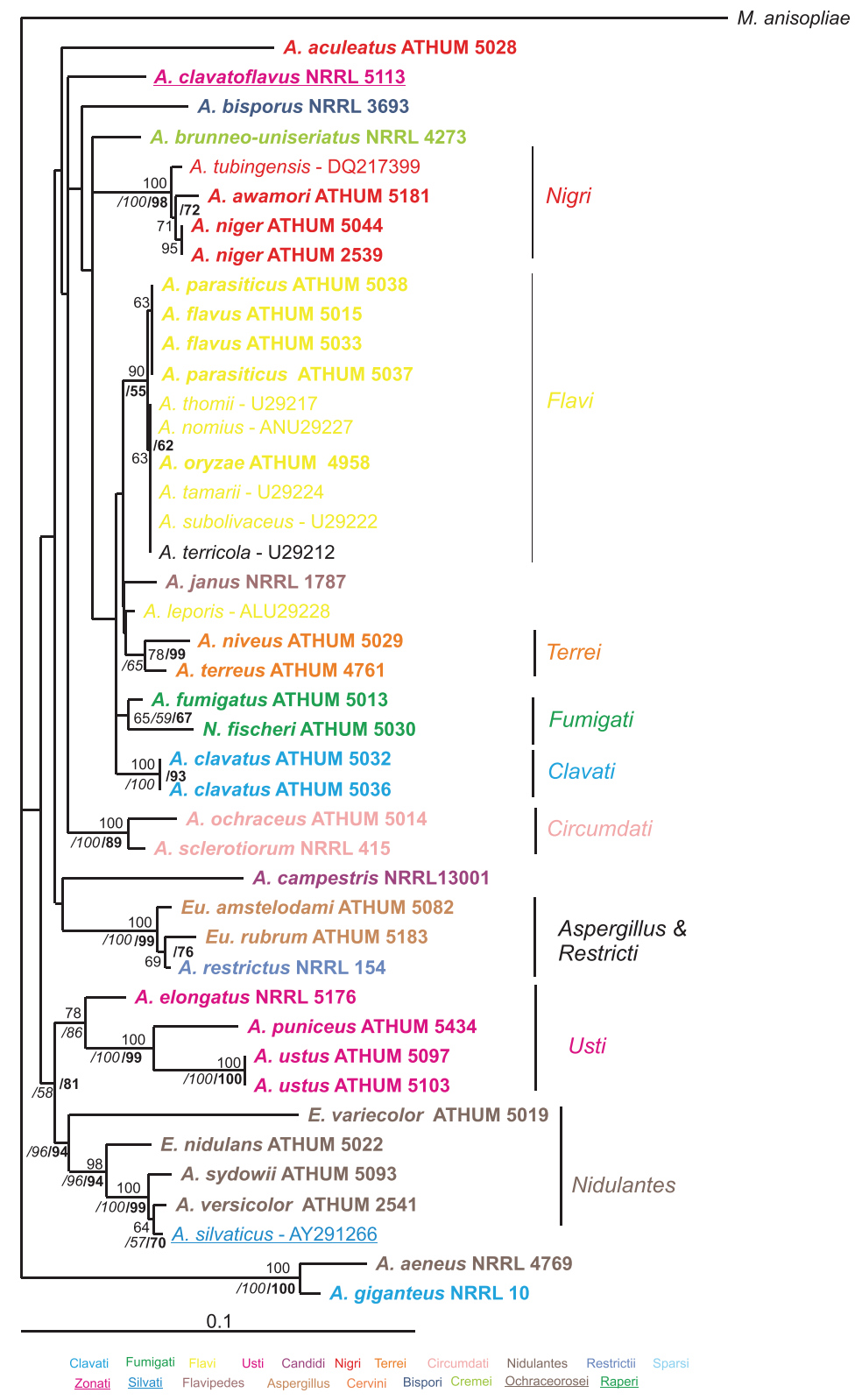

Figure 5: Phylogenetic tree constructed from unambiguously aligned combined DNA sequences of the mitochondrial $r n s$ gene as produced by NJ. Sequences obtained during this study are presented in bold, whereas those retrieved from GenBank are shown in roman with their Accession Numbers. Clade credibility using NJ calculated from 1,000 replicates (numbers in roman), parsimonial BS support calculated from 100 replicates (numbers in italics) using PAUP, and PPs produced by 1,000,000 generations (numbers in bold) using MrBayes are shown.

Eurotium halophilicum are excluded) and Flavi (A. alliaceus and $A$. leporis are excluded) presented higher than $90 \%$ support for at least two different approaches of bootstrap analyses, whereas sections Clavati, Cremei, Fumigati, and Sparsi clustered with excellent support (>90\%) for one methodology and at least good (51-89\%) for the other two (Figure 2). Species belonging to sections Nigri, Nidulantes, and Usti were divided into two well discriminated, well supported groups in each case (Figure 2). Subgenus Nidulantes based on this dataset seems to be polyphyletic; that is, sections Nidulantes, Raperi, Silvati, and Usti form a single cluster, but sections Bispori, Ochraceorosei, and Sparsi are scattered. Finally, strains from different sections, that is, A. bisporus NRRL 3693 (Bispori), A. brunneouniseriatus NRRL 4273 (Cremei), A. aeneus NRRL 4769 (Nidulantes) and $A$. paradoxus AY373860 (Clavati) formed a mixed cluster (Figure 2). This cluster grouped as a sister clade to a group consisting of $A$. crystallinus and $A$. malodoratus (see Figure 2) with excellent NJ, MP and PP (100\%) support.

All ben A amplicons contained three introns with the exception of Emericella variecolor and A. versicolor which lack the last intron. As shown in Figure 3, the single tree 


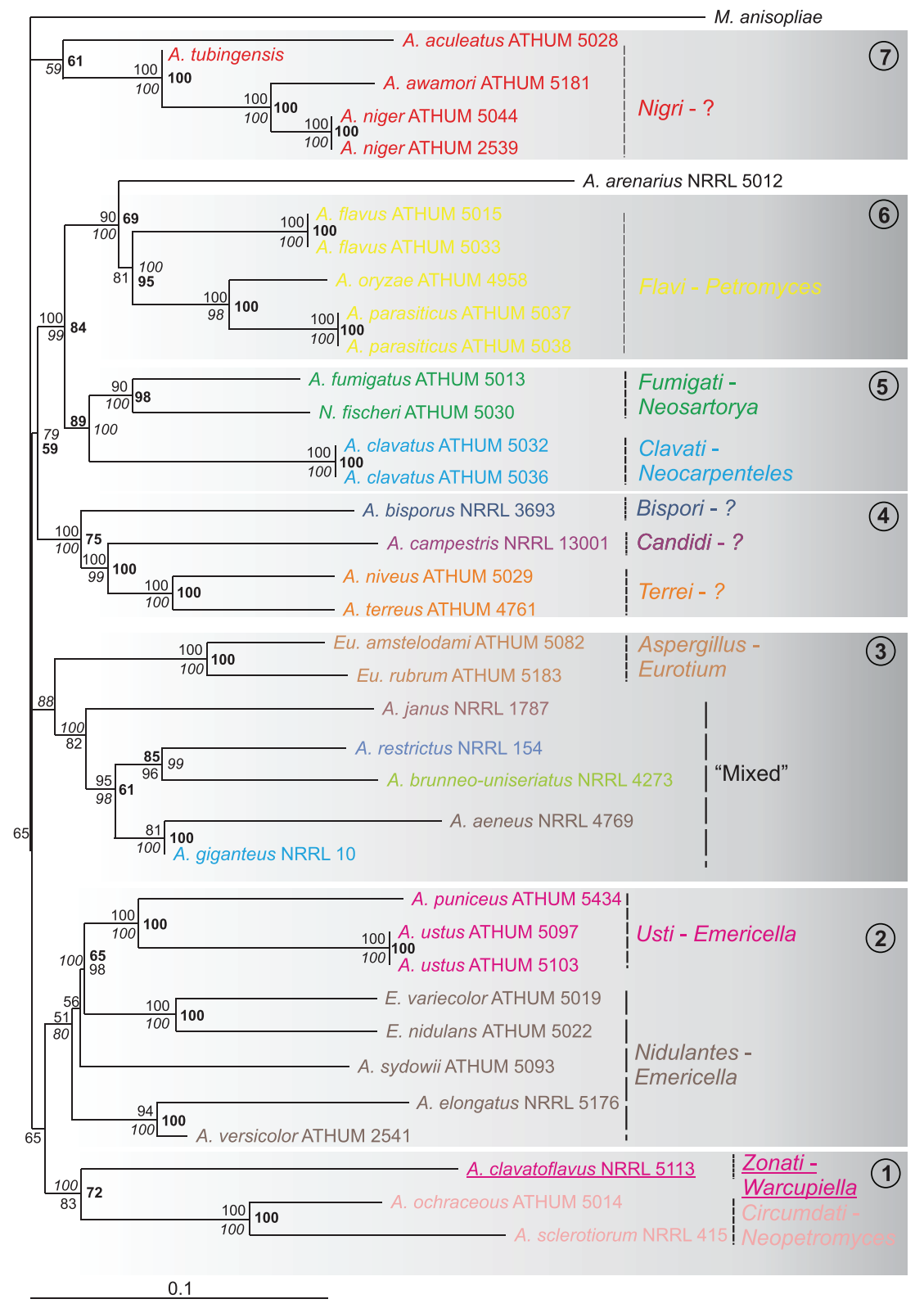

FIGURE 6: Phylogenetic tree constructed from unambiguously aligned concatenated DNA sequences of the nuclear ITS, IGS, benA, and $r p b 2$ genes and the mitochondrial coxl and rns genes as produced by NJ. Sequences obtained during this study are presented in bold, whereas those retrieved from GenBank are shown in roman with their Accession Numbers. Clade credibility using NJ calculated from 1,000 replicates (numbers in roman), parsimonial BS support calculated from 100 replicates (numbers in italics) using PAUP, and PPs produced by 1,000,000 generations (numbers in bold) using MrBayes are shown. Solid lines with numbers in circles represent the seven clusters, while the dotted lines show the sections of the species examined and their teleomorphs.

based on ben A nucleotide sequences discriminated clearly species that belong to sections Candidi, Cervini, Circumdati, Clavati, Flavi, Fumigati, Nigri, Raperi and Terrei. Topology support was extremely good for these sections with at least one methodology showing $100 \%$ bootstrap value in all cases (except section Sparsi: 98, 92, and 62\% for NJ, MP bootstrap and PP, resp.). As in the case of the ITS dataset section Nigri was divided into two groups with the second group containing five members (i.e., A. aculeatus, A. aculeatinus,
A. bahamensis, A. homomorphus, and A. uvarum; Figure 3). Species within sections Aspergillus, Cremei, Nidulantes, Penicillium, Usti, and Restricti although they showed good clustering, they failed to produce high bootstrap support due to the odd discrepancies and must therefore be approached more cautiously.

In accordance with ITS and $b e n \mathrm{~A}$, analyses of the $r p b 2$ dataset provided similar or better phylogeny (Figure 4) for species of sections Aspergillus, Clavati, Cervini, Circumdati, 
Fumigati, Penicillium, Raperi, and Restricti grouping with excellent bootstrap support (93-100\%, irrelevant to the bootstrap methodology applied). Here again, section Nigri species were placed into two distinct groups with bootstrap support of $100,100,99 \%$ and $77,56,98 \%$ (for the NJ and MP bootstrap and $\mathrm{PP}$ of the BI analyses of groups 1 and 2, resp.), but the two groups were sister clades, supported with 69,56 , and $89 \% \mathrm{NJ}$ and MP bootstrap and PP values, with the exception of $A$. japonicus, which was placed basally to rest of the Nigri species. The phylogenies found after the analyses of IGS (Supplemental Figure S1) and cox1 (Supplemental Figure S2) did not improve the picture drawn from ITS, benA, and $r p b 2$ trees but instead added some ambiguities which could be attributed mainly to the limited number of species examined since this may inhibit the good resolution of the species phylogenetic relationships. Nevertheless, phylogenies from both IGS and coxl can be used for better distinguishing species within the same section (e.g., A. niger with A. awamori), as also found in a recent paper with other genes by Perrone et al. [39], but are not suitable to discriminate the sections themselves. Finally, the tree produced by the mitochondrial $r n s$ dataset showed very good clustering of species belonging to sections Aspergillus, Clavati, Circumdati, Nigri, Nidulantes, and Usti (with the exception of $A$. elongatus which is basal to the other Usti species) with excellent bootstrap support (values range from 93 to 100\%; Figure 5) and secondarily weaker bootstrap values of $55-90 \%$ for species that belong to Flavi, Terrei, and Fumigati (Figure 5). Thus, a general conclusion from single gene analyses is that subgenera Aspergillus, Candidi, Fumigati, and Terrei evidently form well supported clusters, while sections of the subgenera Circumdati, and Nidulantes are dispersed.

To achieve full exploitation of the information obtained from nuclear and mitochondrial genes, a tree was constructed based on the combined gene datasets. The concatenated dataset included 4,507 characters, with 2,217 parsimony informative characters and parsimony analysis provided a single tree. The tree length was based on 13,497 steps (CI: 0.40, HI: 0.60 , RI: 0.52, RC: 0.21). Analysis of the same dataset with NJ and BI methods produced similar trees with identical topologies wherever there was a strong support (Figure 6). The sections that contained the larger numbers of strains examined, that is, Flavi, Nigri, Nidulantes, along with sections Aspergillus, Circumdati, Clavati, Fumigati, and Terrei, were well discriminated with better support compared to the results produced by single gene analyses (Figure 6). In addition, the tree produced by the concatenated dataset further supports the polyphyly of subgenus Circumdati, as single gene phylogenies have also shown, since sections Nigri, Flavi, and Circumdati form separate clades at the base of the tree. The section Circumdati, represented in this study by species $A$. ochraceus and $A$. sclerotiorum, forms a sister clade to section Zonati, represented by A. clavatoflavus. Both sections can be a sibling clade to section Nidulantes based only on the NJ analysis. Both MP and BI analyses show that this relation is not supported and thus sections Circumdati and Zonati collapse at the base of the tree.

Most importantly, the tree of the concatenated dataset presented a backbone of seven major clades which could be related to the developmental patterns of the teleomorphs (Figure 6). In the subgenus Circumdati, clade 1 includes members of section Circumdati with connection to Neopetromyces [40], a genus with light-coloured multilocular ascostromata. It is distantly related to section Flavi (clade 6), with a recently confirmed connection to Petromyces [41, 42], characterized by black multilocular ascostromata and section Nigri (clade 7) with unknown teleomorphs, even though sclerotia are formed that should be considered as ascomatal primordia [43]. In the subgenus Nidulantes, the sections Nidulantes, and Usti in clade 2 cluster with Emericella teleomorphs that produce ascomata surrounded by Hülle cells. Additionally, few species belonging to subgenus Nidulantes are in clade 3 which forms a cluster intermixed with subgenus Aspergillus sections Aspergillus and Restricti that contain Eurotium teleomorphs with thin layered ascomata loosely suspended in the mycelium. In the subgenus Fumigati, in clade 5, the section Fumigati is connected to Neosartorya with ascomata composed of abundant sterile hyphae and the section Clavati is connected to Neocarpentelles with unilocular stromatic ascomata. Clade 4 includes taxa in sections Terrei, Candidi, and Bispori without evident teleomorphic connections.

\section{Discussion}

Fungal phylogeny based solely on morphological criteria or on single genes like the rRNA gene sequences may not always elucidate the taxonomic status of the organisms examined [14]. Also, studies based on a single gene do not always faithfully represent the history of the entire genome containing it and comparisons may give the wrong conclusions about the relationship of a fungus with members of the same species or even the same genus [44]. Certainly, the nuclear $r D N A$ repeat is the most popular region in molecular phylogenetic studies because it is multicopied and contains the highly conserved genes $18 \mathrm{~S}$, 5.8S, and $28 \mathrm{~S}$, as well as the variable domains of ITS1 and ITS2, and the nontranscribed IGS region. In addition, the $18 \mathrm{~S}$ and $28 \mathrm{~S}$ regions often harbour group I introns, inserted at highly conserved positions, contributing further information in regard to this region. These properties have been exploited at length in studies of Aspergillus species $[11,15,45]$ or other fungal genera $[28,46,47]$. In a similar way, the mtDNA of fungi has lately attracted considerable interest as an alternative or complementary molecule for phylogenetic studies $[26,35,48]$, because of its high copy numbers, richness in AT sequences, lack of methylation, universal gene functions, highly conserved regions, as well as variable domains and introns [49]. Currently, the most common mt genes used in fungal phylogenetic studies are rns and coxl, because the primers designed for these genes can be applied to a wide range of taxonomically different fungi $[50,51]$. Our analyses of representative species from the most common sections of Aspergillus showed for the first time here that mitochondrial based phylogenies indicate different evolutionary pathways which may provide valuable data for taxonomic purposes when combined with morphological and nuclear based molecular datasets. 
It has often been argued that the reconstruction of phylogenies is better achieved by multiple datasets, not only of nuclear but also of mitochondrial origin [52]. In the present study, the ITS and IGS regions from the nu-rRNA gene complex and rns and cox 1 from the mt genomes were chosen to study the phylogenetic relationships of Aspergillus species. To overcome possible ambiguities raised by the analyses of these genes, data from the nuclear genes $r p b 2$ and ben $\mathrm{A}$ that are generally accepted as phylogenetically informative were also used $[5,45,53]$. Although benA sequences were excluded from the concatenated data used in the most recent multigene approach, since the presence of two or three introns in the corresponding ben $\mathrm{A}$ amplicons was attributed to amplification of paralogous genes [23], we have chosen to use this data in our work for three reasons: (a) because 34 out of the 36 species examined contained all three introns, only two species were missing one intron, (b) the identity levels of the 34 amplicon sequences (i.e., containing all three introns) was $>84 \%$, and (c) exon identity between all 36 amplicons ranged between 86 and $96 \%$. As shown in results, this choice was justified because the ben $\mathrm{A}$ based phylogenetic tree provided additional information as, for example, in the case of members of section Nigri that were clustered together but clearly differentiated the uniseriate from the biseriate species. Thus, all data from nuclear and mt genes were combined as a single unit in order to blend information from two independent heritage lines, to examine whether this approach provides useful information in resolving phylogenetic ambiguities within the genus Aspergillus.

The single gene trees obtained from ITS, IGS, $r p b 2$, and rns and, mainly, the concatenated datasets, strengthen the fraternisation of Clavati to subgenus Fumigati, as species of section Clavati form a sister group to species within section Fumigati, with MP bootstrap and PP support that reached 89 and $100 \%$, respectively, at the combined dataset (Figure 6). This is in full accordance with results obtained from a recent multilocus analysis that was based solely on nuclear genes and the addition of section Cervini species in this subgenus $[23,24]$. Interestingly enough, our analysis based on the ITS dataset showed that although sufficient bootstrap or posterior probability support was lacking, uniseriate species that belong to subgenera Fumigati and Aspergillus clustered together, and apart from other species of Aspergillus that are biseriate (Figure 2).

Section Usti is of particular interest since its elimination and the transfer of species A. ustus, A. deflectus, A. puniceus, A. granulosus, and A. pseudodeflectus to section Nidulantes, and species $A$. conjunctus, A. funiculosus, A. silvaticus, A. panamensis and $A$. anthodesmis to section Sparsi was initially proposed [15], only to be rejected a few years later, by exploiting the results of polyphasic approaches in which the sequences of the nuclear $\beta$-tubulin, calmodulin, actin, and ITS genes were used $[5,18]$. These works not only preserved section Usti but also added eight more species in it, namely, A. ustus, A. puniceus, A. granulosus, A. pseudodeflectus, A. calidoustus, A. insuetus, A. kevei, and Emericella heterothallica. Finally, more recent works added nine more Aspergillus species in it, seven and two, respectively, bringing the total number to $21[11,24]$. In full agreement with these, our results suggest that section Usti should be retained as an independent section within the subgenus Nidulantes with further support, since both the ITS and concatenated data analyses show that species of Usti, Nidulantes, Silvati, and Raperi comprise a single clade supported (e.g., from ITS dataset) by NJ-MP bootstrap (51\% and 71\%, resp.), and BI Posterior Probability (95\%), and thus, altogether may be considered as one group within subgenus Nidulantes (Figures 2 and 6).

Until now, several genes have been proposed for the identification of species within the section of black aspergilli. However, the results obtained are very variable. IGS and cox 1 sequences were found unsuitable to discriminate species within this section (Supplemental Figures S1 and S2), because they either exhibited too high intraspecies variability or this variability was also extended to inter-species comparisons $[3,20]$. Similarly, ITS sequences could distinguish four groups within the $A$. niger species complex but failed to do so for species like $A$. carbonarius and $A$. sclerotioniger, or A. japonicus, A. aculeatus and A. uvarum which had identical ITS sequences [20]. Also, mt cytochrome b (cob) gene sequences were informative for many species within the Nigri section but they failed to distinguish between $A$. niger and $A$. tubingensis [54]. Thus, the best discriminatory results based on single gene sequences were those obtained from $\beta$-tubulin and calmodulin phylogenetic analysis which placed 26 taxa within subgenus Circumdati section Nigri, further dividing them into 5 main clades, grouping all uniseriate species (7) in only one clade (the $A$. aculeatus clade) $[13,45]$. In the present work, single gene datasets with many individual sequences (ITS, ben A, and $r p b 2$ ) and the concatenated dataset clearly distinguished members of Nigri and divided them into two groups: the first containing $A$. aculeatus, $A$. aculeatinus, $A$. bahamensis, A. uvarum-in all datasets-and A. homomorphus, A. japonicus - wherever data were available-, and the second composed of all the rest species (see e.g., ITS or benA trees, Figures 2 and 3). It is interesting to note that these two clades correspond to the uniseriate and biseriate conidial head formation, which may be a possible explanation why previous studies that did not take into account this characteristic, failed to cluster them accordingly [13, 20, 45]. An additional explanation for the splitting of section Nigri species into two subgroups may be attributed to the range of variability resulting from adaptation of the species to the Mediterranean type ecosystem. This is also evident by the variability found in the morphological characteristics of the Greek Aspergillus isolates (Table 2). The biseriate species within section Nigri cluster with good bootstrap support (67\% bootstrap for NJ and $84 \%$ PP) with biseriate species belonging to sections Flavipedes, Terrei, Ochraceorosei, and Sparsi, while the uniseriate species of Nigri are basal to the above-mentioned biseriate clades of sections Flavi with good support (70\% PP, Figure 2). Thus, the differentiation of Nigri species studied here into uniseriate and biseriate not only helped to group them in combination with molecular markers into two clearly distinct clades, but in addition clustered together biseriate species of all sections.

Species of the Flavi section were grouped well together, with high bootstrap support ( $>80 \%)$, irrelevant to the dataset used. While single gene analysis, like ben $\mathrm{A}$, failed to separate 
A. flavus from A. oryzae, the concatenated dataset clearly demonstrated its advantages. It could fully discriminate all species examined and could also confirm that taxa within the section, like $A$. oryzae, A. flavus, and A. parasiticus are phylogenetically distantly related to $A$. ochraceus, as previously proposed [55] and also recently shown with the polyphyly of A. flavus, and in extent of section Flavi [56].

Previous reports showed that sections Circumdati, Flavi and Nigri do not cluster together [23] and this was further confirmed by our phylogenetic analyses of ITS, benA, and $r p b 2$ datasets. However, it must be pointed out that this is in contradiction with our morphological observations which demonstrated significant morphological similarities, that is, biseriate conidial heads, large globose vesicles, ascostromata when teleomorph present, and suggest a possible common origin of these sections. The latter is further supported by the analysis of the mt rns sequences, even though the bootstrap values do not seem significant enough. Thus, this intriguing controversy between molecular data and morphological observations underlines the necessity to use polyphasic approaches in one hand and increase the number of species examined on the other in order to solve difficult taxonomy problems.

The single tree produced by our concatenated dataset showed that although $A$. niveus and $A$. terreus, which belong to section Terrei, are closely related and with excellent support (100\% irrelevant of the method applied), they are far apart from A. janus (section Flavipedes) and A. arenarius, both of which were previously placed closely to species of Terrei, Flavipedes, and Candidi [23]. This causes some concern because our dataset places $A$. arenarius as a sister clade to section Flavi with good support (bootstrap of $90 \%$ for NJ and 100\% for MP analyses; 69\% Posterior Probabilities for the BI analyses; Figure 6), contrary to suggestions that it is unrelated to any section of Aspergillus [24]. Similarly, our concatenated analysis contradicts the proposed merger of sections Terrei and Flavipedes [24, 57] because A. janus is excluded from being a sister clade of section Terrei (Figure 6). Since the datasets from the other genetic loci, examined in this work, show a random positioning of $A$. janus and $A$. arenarius, it is obvious that a thorough molecular analysis of the relations between Terrei and Flavipedes is urgently needed, especially if previous members of Versicolores, like $A$. janus and $A$. arenarius, are to be included within these sections. Recently, Jurjevic et al. [58] undertook the study of section Versicolores and provided useful phylogenetic data on species of this section based on multilocus DNA phylogeny, but still the inclusion of these species in this section has not been resolved.

As for the subgenus Nidulantes, the tree based on concatenated data showed that it is a compact assemblage of species from sections Nidulantes and Usti, as expected [23, 24]. Finally, the intermixed cluster of five species from several sections that was not resolved by the analysis of the six genetic loci used in our work is another interesting area of research that will certainly require the introduction of either more genes or more taxa, as it has already been done in other studies facing similar problems $[3,52]$.
In conclusion, our study clearly shows that all molecules used contributed to the resolution of phylogenetic relationships in Aspergillus, irrespective to the different ways that the phylogenetic data were processed. It also confirms that single gene based analyses do not solve all ambiguities and do not always represent the evolutionary history of the species. Certainly, Aspergillus is a very diverse genus and it is not always easy to determine the phylogenetic relationships between species. Thus, the combination of sequence information from nuclear and mitochondrial genes resolves several circumscriptions and relationships of Aspergillus species within different sections and demonstrates the advantages of this multigenic approach. It is expected that the vast amount of information derived from the released complete genomes of Aspergillus species will be utilized in future studies by many researchers to select new additional genes from both (nuclear and mitochondrial) evolutionary lines. Such combined data is expected to provide the appropriate levels of resolving power in future phylogenetic studies.

\section{Acknowledgments}

The authors are greatly indebted to Dr. Stephen Peterson, Peoria, USA, for providing ex-type strains of Aspergillus spp. This research was supported by the Greek General Secretariat of Research and Technology (Project TR-5) in the frame of the "Competitiveness" Program of European Commission.

\section{References}

[1] V. Meyer, B. Wu, and A. F. J. Ram, "Aspergillus as a multipurpose cell factory: current status and perspectives," Biotechnology Letters, vol. 33, pp. 469-476, 2011.

[2] D. Lubertozzi and J. D. Keasling, "Developing Aspergillus as a host for heterologous expression," Biotechnology Advances, vol. 27, no. 1, pp. 53-75, 2009.

[3] D. M. Geiser, M. A. Klich, J. C. Frisvad, S. W. Peterson, J. Varga, and R. A. Samson, "The current status of species recognition and identification in Aspergillus," Studies in Mycology, vol. 59, pp. 1-10, 2007.

[4] J. C. Frisvad, T. O. Larsen, R. de Vries et al., "Secondary metabolite profiling, growth profiles and other tools for species recognition and important Aspergillus mycotoxins," Studies in Mycology, vol. 59, pp. 31-37, 2007.

[5] S. A. Balajee, J. Houbraken, P. E. Verweij et al., "Aspergillus species identification in the clinical setting," Studies in Mycology, vol. 59, pp. 39-46, 2007.

[6] P. Krijgsheld, R. Bleichrodt, G. J. van Veluw et al., "Development in Aspergillus," Studies in Mycology, vol. 74, pp. 1-29, 2013.

[7] B. K. Raper and D. I. Fennell, The Genus Aspergillus, The Williams and Wilkins, Baltimore, Md, USA, 1965.

[8] W. Gams, M. Christensen, A. H. S. Onions, J. I. Pitt, and R. A. Samson, "Infrageneric taxa of Aspergillus," in Advances in Penicillium and Aspergillus Systematics, R. A. Samson and J. I. Pitt, Eds., pp. 55-62, Plenum Press, New York, NY, USA, 1985.

[9] J. I. Pitt, R. A. Samson, and J. C. Frisvad, "List of accepted species and their synonyms in the family Trichocomaceae," in Integration of Modern Taxonomic Methods For Penicillium and Aspergillus Classification, R. A. Samson and J. I. Pitt, Eds., 
pp. 9-49, Harwood Academic Publishers, Amsterdam, The Netherlands, 2000.

[10] R. A. Samson and J. Varga, "What is a species in Aspergillus?" Medical Mycology, vol. 47, supplement 1, pp. S13-S20, 2009.

[11] R. A. Samson, J. Varga, M. Meijer, and J. C. Frisvad, "New taxa in Aspergillus section Usti," Studies in Mycology, vol. 69, pp. 81-97, 2011.

[12] R. A. Samson, S. W. Peterson, J. C. Frisvad, and J. Varga, "New species in Aspergillus section Terrei," Studies in Mycology, vol. 69, pp. 39-55, 2011.

[13] J. Varga, J. C. Frisvad, S. Kocsubé et al., "New and revisited species in Aspergillus section Nigri," Studies in Mycology, vol. 69, pp. 1-17, 2011.

[14] M. L. Berbee, D. A. Carmean, and K. Winka, "Ribosomal DNA and resolution of branching order among the Ascomycota: how many nucleotides are enough?" Molecular Phylogenetics and Evolution, vol. 17, no. 3, pp. 337-344, 2000.

[15] S. W. Peterson, "Phylogenetic relationships in Aspergillus based on rDNA sequence analysis," in Integration of Modern Taxonomic Methods for Penicillium and Aspergillus Classification, R. A. Samson and J. I. Pitt, Eds., pp. 323-355, Harwood Academic Publishers, Amsterdam, The Netherlands, 2000.

[16] M. L. Berbee, "The phylogeny of plant and animal pathogens in the Ascomycota," Physiological and Molecular Plant Pathology, vol. 59, no. 4, pp. 165-187, 2001.

[17] P. J. Keeling, M. A. Luker, and J. D. Palmer, "Evidence from betatubulin phylogeny that microsporidia evolved from within the fungi," Molecular Biology and Evolution, vol. 17, no. 1, pp. 23-31, 2000.

[18] J. Houbraken, M. Due, J. Varga, M. Meijer, J. C. Frisvad, and R. A. Samson, "Polyphasic taxonomy of Aspergillus section Usti," Studies in Mycology, vol. 59, pp. 107-128, 2007.

[19] R. A. Samson, S. Hong, S. W. Peterson, J. C. Frisvad, and J. Varga, "Polyphasic taxonomy of Aspergillus section Fumigati and its teleomorph Neosartorya," Studies in Mycology, vol. 59, pp. 147203, 2007.

[20] R. A. Samson, P. Noonim, M. Meijer, J. Houbraken, J. C. Frisvad, and J. Varga, "Diagnostic tools to identify black aspergilli," Studies in Mycology, vol. 59, pp. 129-145, 2007.

[21] J. Varga, M. Due, J. C. Frisvad, and R. A. Samson, “Taxonomic revision of Aspergillus section Clavati based on molecular, morphological and physiological data," Studies in Mycology, vol. 59, pp. 89-106, 2007.

[22] J. Varga, J. C. Frisvad, and R. A. Samson, "Polyphasic taxonomy of Aspergillus section Candidi based on molecular, morphological and physiological data," Studies in Mycology, vol. 59, pp. 7588, 2007.

[23] S. W. Peterson, "Phylogenetic analysis of Aspergillus species using DNA sequences from four loci," Mycologia, vol. 100, pp. 205-226, 2008.

[24] S. W. Peterson, J. Varga, J. C. Frisvad, and R. A. Samson, "Phylogeny and subgeneric taxonomy of Asperillus," in Aspergillus in the Genomic Era, J. Varga and R. A. Samson, Eds., pp. 33-56, Wageningen Academic Publishers, Wageningen, The Netherlands, 2008.

[25] V. N. Kouvelis, D. V. Ghikas, S. Edgington, M. A. Typas, and D. Moore, "Molecular characterization of isolates of Beauveria bassiana obtained from overwintering and summer populations of Sunn Pest (Eurygaster integriceps)," Letters in Applied Microbiology, vol. 46, no. 3, pp. 414-420, 2008.
[26] V. N. Kouvelis, A. Sialakouma, and M. A. Typas, "Mitochondrial gene sequences alone or combined with ITS region sequences provide firm molecular criteria for the classification of Lecanicillium species," Mycological Research, vol. 112, no. 7, pp. 829844, 2008.

[27] M. P. Pantou, O. K. Strunnikova, V. Y. Shakhnazarova, N. A. Vishnevskaya, V. G. Papalouka, and M. A. Typas, "Molecular and immunochemical phylogeny of Verticillium species," Mycological Research, vol. 109, no. 8, pp. 889-902, 2005.

[28] M. P. Pantou, A. Mavridou, and M. A. Typas, "IGS sequence variation, group-I introns and the complete nuclear ribosomal DNA of the entomopathogenic fungus Metarhizium: excellent tools for isolate detection and phylogenetic analysis," Fungal Genetics and Biology, vol. 38, no. 2, pp. 159-174, 2003.

[29] M. A. Klich and J. I. Pitt, A LaboraTory Guide to Common Aspergillus Species and Their Teleomorphs, CSIRO, North Ryde, Australia, 1988.

[30] M. A. Typas, A. M. Griffen, B. W. Bainbridge, and J. B. Heale, "Restriction fragment length polymorphisms in mitochondrial DNA and ribosomal RNA gene complexes as an aid to the characterization of species and sub-species populations in the genus Verticillium," FEMS Microbiology Letters, vol. 95, no. 2-3, pp. 157-162, 1992.

[31] B. Henrion, F. Le Tacon, and F. Martin, "Rapid identification of genetic variation of ectomycorrhizal fungi by amplification of ribosomal RNA genes," New Phytologist, vol. 122, pp. 289-298, 1992.

[32] Y. J. Liu, S. Whelen, and B. D. Hall, "Phylogenetic relationships among ascomycetes: evidence from an RNA polymerase II subunit," Molecular Biology and Evolution, vol. 16, pp. 1799$1808,1999$.

[33] N. L. Glass and G. C. Donaldson, "Development of primer sets designed for use with the PCR to amplify conserved genes from filamentous ascomycetes," Applied and Environmental Microbiology, vol. 61, no. 4, pp. 1323-1330, 1995.

[34] T. J. White, T. Bruns, S. Lee, and J. W. Taylor, "Amplification and direct sequencing of fungal ribosomal RNA genes for phylogenetics," in PCR ProTocols, M. A. Innis, D. H. Gelfand, J. J. Sninsky, and T. J. White, Eds., pp. 315-322, Academic Press, San Diego, Calif, USA, 1990.

[35] D. V. Ghikas, V. N. Kouvelis, and M. A. Typas, "The complete mitochondrial genome of the entomopathogenic fungus Metarhizium anisopliae var. anisopliae: gene order and trn gene clusters reveal a common evolutionary course for all Sordariomycetes, while intergenic regions show variation," Archives of Microbiology, vol. 185, no. 5, pp. 393-401, 2006.

[36] D. L. Swofford, PAUP*. Phylogenetic Analysis Using Parsimony $\left({ }^{*}\right)$ and Other Methods, Version 4, Sinauer Associates, Sunderland, Mass, USA, 1998.

[37] J. P. Huelsenbeck, MrBayes: Bayesian Inference of Phylogeny (Software), University of Rochester, New York, NY, USA, 2000.

[38] Z. Yang, "PAML: a program package for phylogenetic analysis by maximum likelihood," CABIOS, vol. 13, pp. 555-556, 1997.

[39] G. Perrone, G. Stea, F. Epifani, J. Varga, J. C. Frisvad, and R. A. Samson, "Aspergillus niger contains the cryptic phylogenetic species A. awamori," Fungal Biology, vol. 115, pp. 1138-1150, 2011.

[40] J. C. Frisvad and R. A. Samson, "Neopetromyces gen. nov. and an overview of teleomorphs of Aspergillus subgenus Circumdati," Studies in Mycology, vol. 2000, no. 45, pp. 201-207, 2000.

[41] B. W. Horn, G. G. Moore, and I. Carbone, "Sexual reproduction in Aspergillus flavus,” Mycologia, vol. 101, pp. 423-429, 2009. 
[42] B. W. Horn, J. H. Ramirez-Prado, and I. Carbone, "The sexual state in Aspergillus parasiticus," Mycologia, vol. 101, pp. 275-280, 2009.

[43] D. M. Geiser, "Sexual structures in Aspergillus: morphology, importance and genomics," Medical Mycology, vol. 47, supplement 1, pp. S21-S26, 2009.

[44] B. F. Lang, M. W. Gray, and G. Burger, "Mitochondrial genome evolution and the origin of eukaryotes," Annual Reviews Genetics, vol. 33, pp. 351-397, 1999.

[45] J. Varga, S. Kocsubé, B. Tóth et al., “Aspergillus brasiliensis sp. nov., a biseriate black Aspergillus species with world-wide distribution," International Journal of Systematic and Evolutionary Microbiology, vol. 57, no. 8, pp. 1925-1932, 2007.

[46] C. Neuveglise, Y. Brygoo, and G. Riba, "28s rDNA group-I introns: a powerful tool for identifying strains of Beauveria brongniartii," Molecular Ecology, vol. 6, no. 4, pp. 373-381, 1997.

[47] C. Wang, L. I. Zengzhi, M. A. Typas, and T. M. Butt, "Nuclear large subunit rDNA group I intron distribution in a population of Beauveria bassiana strains: phylogenetic implications," Mycological Research, vol. 107, no. 10, pp. 1189-1200, 2003.

[48] A. M. Kretzer and T. D. Bruns, "Use of atp6 in fungal phylogenetics: an example from the boletales," Molecular Phylogenetics and Evolution, vol. 13, no. 3, pp. 483-492, 1999.

[49] A. Cambell, J. Mrazek, and s. Karlin, "Genome signature comparisons among prokaryote, plasmid, and mitochondrial DNA," Proceedings of the National Academy of Sciences USA, vol. 96, pp. 9184-9189, 1999.

[50] K. N. Li, D. I. Rouse, and T. L. German, "PCR primers that allow intergeneric differentiation of ascomycetes and their application to Verticillium spp," Applied and Environmental Microbiology, vol. 60, no. 12, pp. 4324-4331, 1994.

[51] X. J. Min and D. A. Hickey, "Assessing the effect of varying sequence length on DNA barcoding of fungi," Molecular Ecology Notes, vol. 7, pp. 365-373, 2007.

[52] A. Rokas and S. B. Carroll, "More genes or more taxa? The relative contribution of gene number and taxon number to phylogenetic accuracy," Molecular Biology and Evolution, vol. 22, no. 5, pp. 1337-1344, 2005.

[53] Y. Tanabe, M. Saikawa, M. M. Watanabe, and J. Sugiyama, "Molecular phylogeny of Zygomycota based on EF-1 $\alpha$ and RPB1 sequences: limitations and utility of alternative markers to rDNA," Molecular Phylogenetics and Evolution, vol. 30, no. 2, pp. 438-449, 2004.

[54] K. Yokoyama, L. Wang, M. Miyaji, and K. Nishimura, "Identification, classification and phylogeny of the Aspergillus section Nigri inferred from mitochondrial cytochrome b gene," FEMS Microbiology Letters, vol. 200, no. 2, pp. 241-246, 2001.

[55] S. Nikkuni, H. Nakajima, S. I. Hoshina et al., "Evolutionary relationships among Aspergillus oryzae and related species based on the sequences of $18 \mathrm{~S}$ rRNA genes and internal transcribed spacers," Journal of General and Applied Microbiology, vol. 44, no. 3, pp. 225-230, 1998.

[56] S. S. Gonçalves, J. F. Cano, A. M. Stchigel et al., "Molecular phylogeny and phenotypic variability of clinical and environmental strains of Aspergillus flavus," Fungal Biology, vol. 116, pp. 11461155, 2012.

[57] J. Varga, B. T. Tóth, S. Kocsubé et al., "Evolutionary relationships among Aspergillus terreus isolates and their relatives," Antonie Van Leeuwenhoek, vol. 88, pp. 141-150, 2005.

[58] Z. Jurjevic, S. W. Peterson, and B. W. Horn, “Aspergillus section Versicolores: nine new species and multilocus DNA sequence based phylogeny," IMA Fungus, vol. 3, pp. 59-79, 2012. 

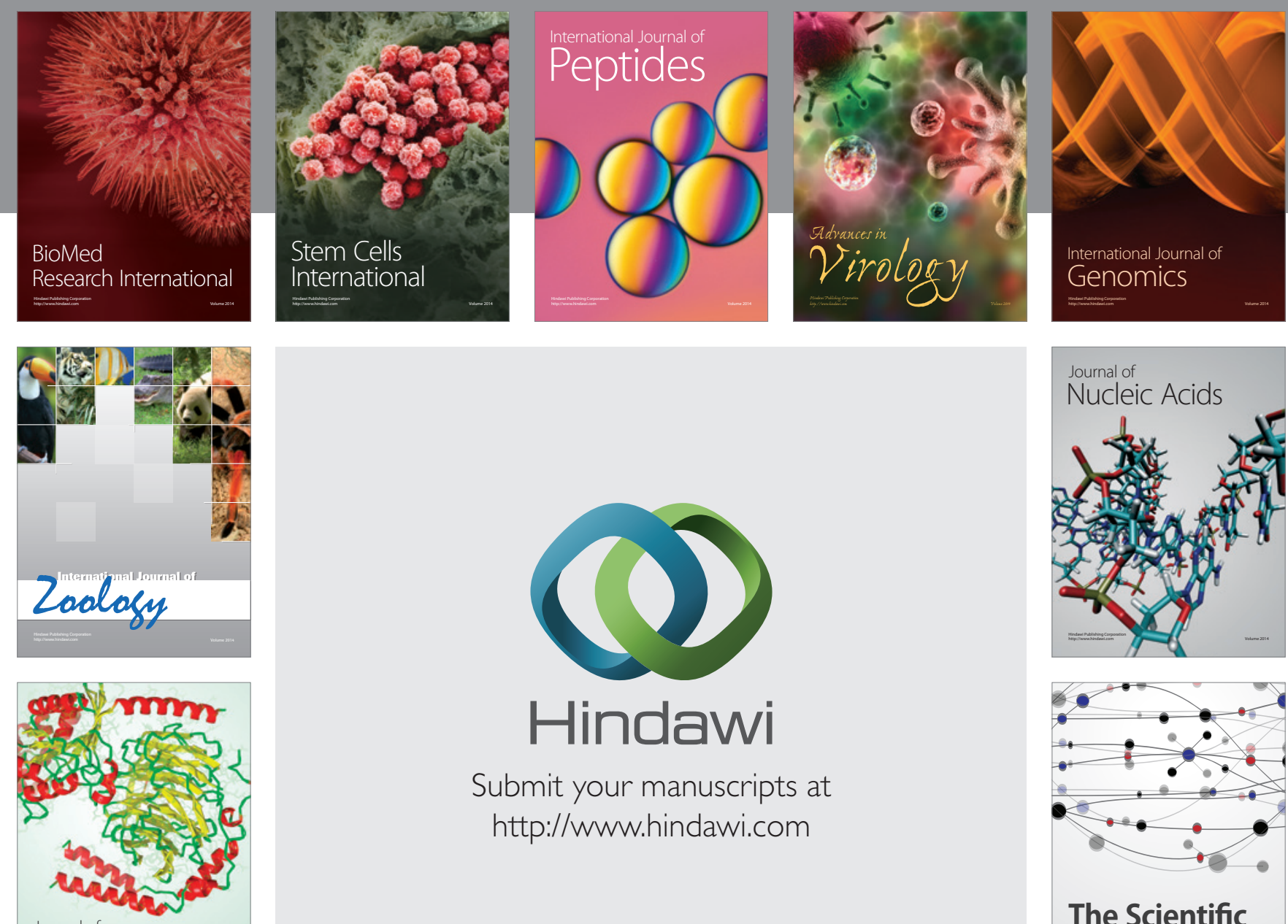

Submit your manuscripts at

http://www.hindawi.com

Journal of
Signal Transduction
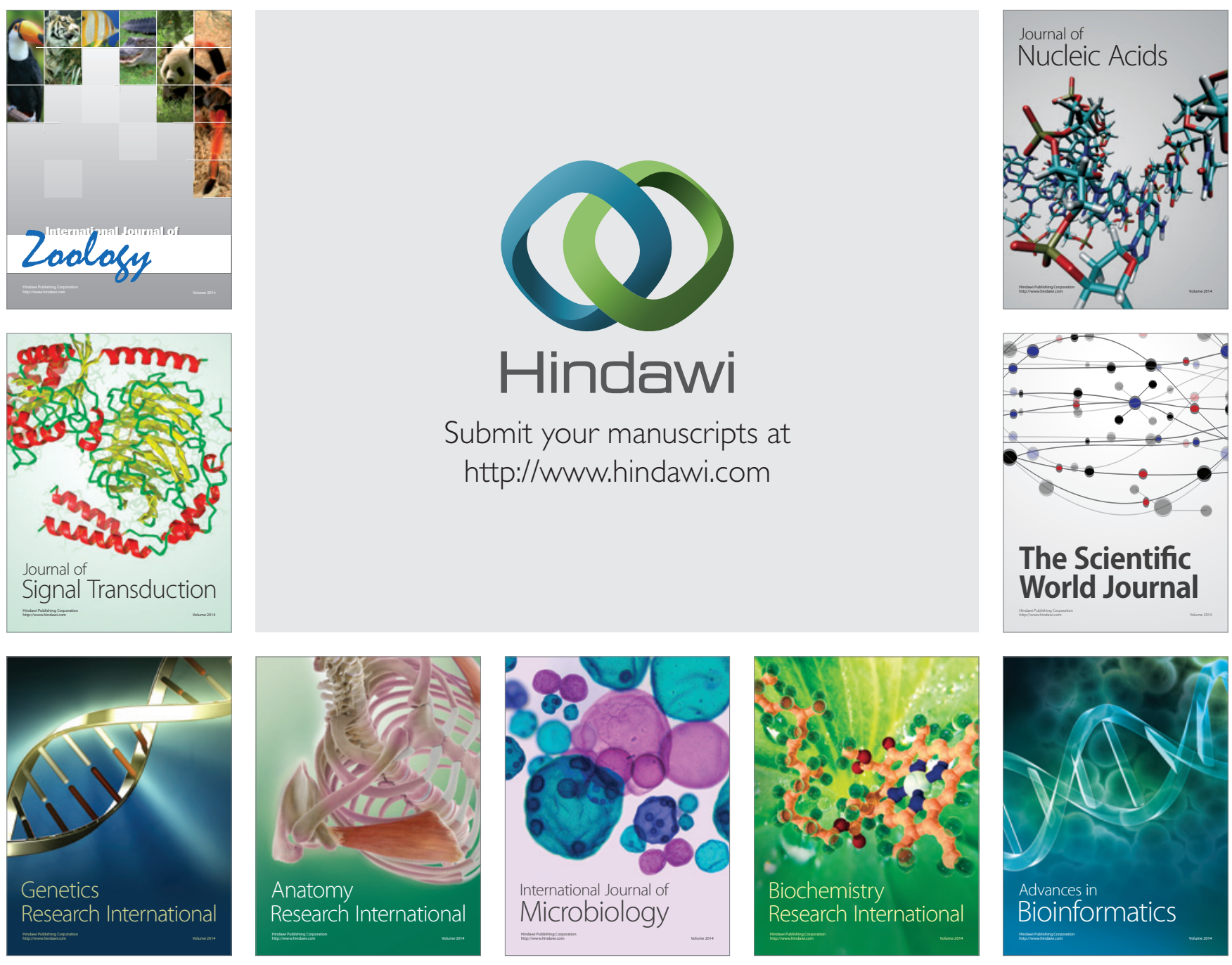

The Scientific World Journal
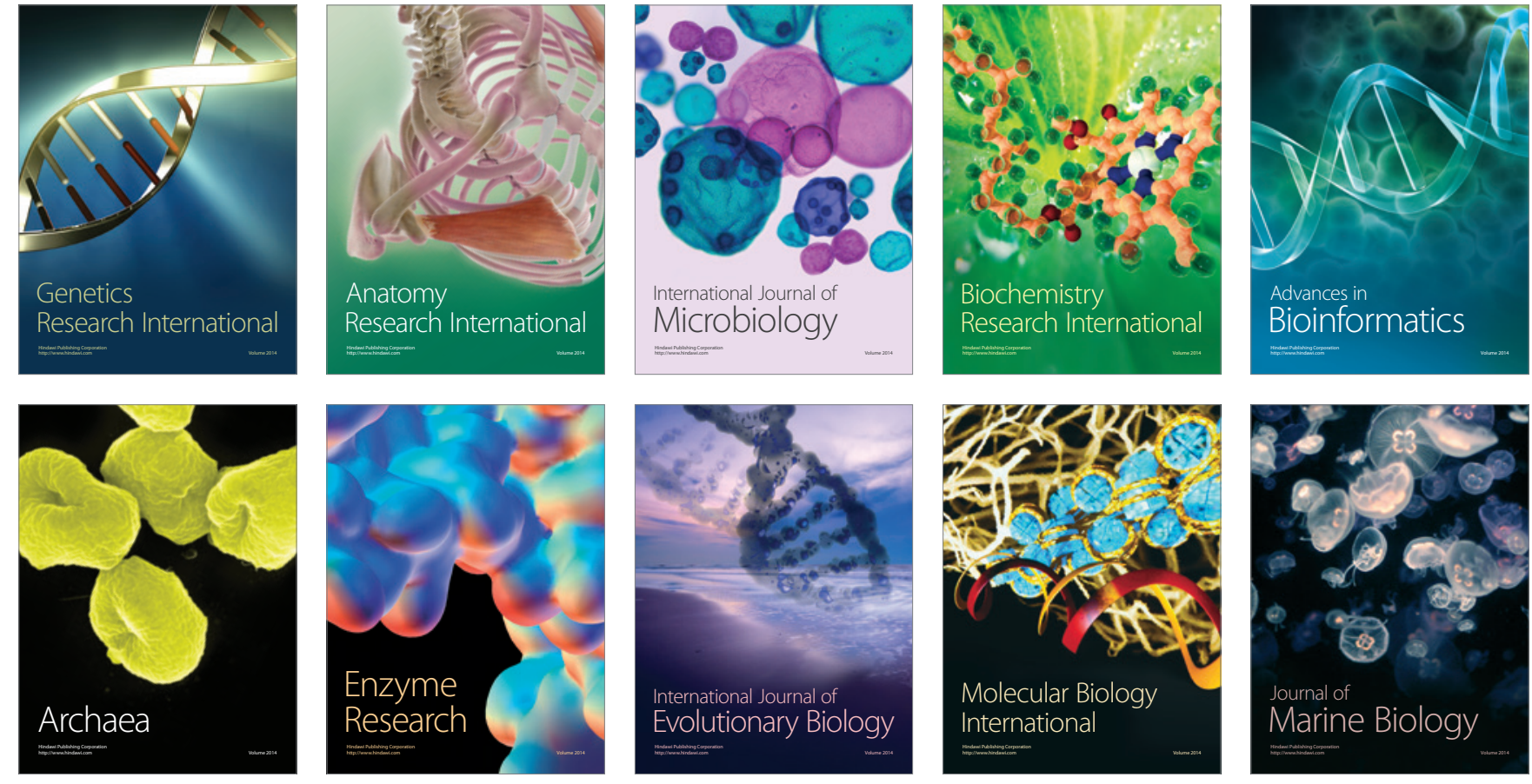